\title{
ILC Higgs Physics Potential
}

\section{Shin-ichi Kawada ${ }^{a, 1, *}$}

${ }^{a} \mathrm{KEK}$,

1-1, Oho, Tsukuba, Ibaraki, 305-0801, Japan

E-mail: skawada@post.kek.jp

Higgs factories based on $e^{+} e^{-}$colliders have the potential to measure the complete profile of the Higgs boson at a level of precision that goes qualitatively beyond the expected capabilities of LHC and HL-LHC. In this contribution, we will review the program of Higgs boson coupling measurements expected from the International Linear Collider, including the most recent updates. These measurements span the range of $e^{+} e^{-}$center-of-mass energies from $250 \mathrm{GeV}$ to $1 \mathrm{TeV}$, and include precision measurements of the Higgs self-coupling.

\footnotetext{
*** Particles and Nuclei International Conference - PANIC2021 ***

*** 5 - 10 September, $2021 * * *$

$* * *$ Online $* * *$

${ }^{1}$ On behalf of ILC IDT (International Development Team) Working Group 3

${ }^{*}$ Speaker
} 


\section{Introduction}

The International Linear Collider (ILC) is one of the proposed future $e^{+} e^{-}$colliders. Its centerof-mass energy $(\sqrt{s})$ starts from $250 \mathrm{GeV}$ which is suitable for the precision Higgs measurements in the Higgsstralhung process $\left(e^{+} e^{-} \rightarrow Z h\right.$ ), but it is upgradable up to $1 \mathrm{TeV}$ enabling access to rare Higgs decays, Higgs self-coupling and BSM signatures in the Higgs sector. Polarized beams will be used at the ILC: $80 \%$ for electrons and $30 \%$ for positrons improving precision of measurements combining different polarization settings. In this contribution, we will discuss the Higgs physics potential at the ILC based on the Ref. [1]. The evaluation of the precision of Higgs measurements is based on the full detector simulation of the International Large Detector (ILD) concept and/or Silicon Detector $(\mathrm{SiD})$ concept [2].

\section{Higgs measurement at the ILC}

In this contribution, we will pick up two examples of the Higgs measurement at the ILC. One of the most important observable is the absolute value of inclusive cross-section $\sigma_{Z h}$ using the recoil mass technique to the $e^{+} e^{-} \rightarrow Z h$ process with $Z \rightarrow e^{+} e^{-} / \mu^{+} \mu^{-} / q \bar{q}$ decay [3, 4]. Since the initial state of the $e^{+} e^{-}$collision is well-known, the mass of the Higgs boson can be determined only by measuring the muon momenta, without looking at any Higgs decay products. This $\sigma_{Z h}$ can be measured with a precision of $0.7 \%$ using $Z \rightarrow e^{+} e^{-} / \mu^{+} \mu^{-}$channels and assuming twenty years running of the ILC [5-7].

Another example of Higgs measurement at the ILC is the measurement of the hadronic decay of the Higgs boson, $h \rightarrow b \bar{b} / c \bar{c} / g g$. The key point of this analysis is the performance of the flavor tagging which directly connects to the separation of $b$-quark and $c$-quark. To perform flavor tagging, a software package LCFIPlus [8] is developed for linear colliders. Its typical performance is shown in Figure 1. Figure 2 shows the 2D templates for $b$-likeness and $c$-likeness for different processes at $\sqrt{s}=500 \mathrm{GeV}$. The processes $h \rightarrow b \bar{b}, h \rightarrow c \bar{c}$, and $h \rightarrow g g$ are nicely separated from each other. After the full event selection, the statistical uncertainties of the cross-section times branching ratio $\sigma \times \mathrm{BR}$ for $h \rightarrow b \bar{b} / h \rightarrow c \bar{c} / h \rightarrow g g$ are estimated to be $0.4 \% / 3.4 \% / 1.5 \%$ with $4 \mathrm{ab}^{-1}$ accumulated luminosity, respectively.

\section{Higgs couplings}

To extract Higgs boson couplings, we use dimension-6 SM Effective Field Theory (EFT) formalism. We use Higgs observables, triple gauge boson coupling observables, and electroweak precision observables as the inputs to the global fit under the EFT framework. We additionally use the ratio of branching ratios from the HL-LHC prospects as the inputs. Details of the precisions of observables and EFT framework can be found in Refs. [1, 10, 11].

Here, we only present the important remarks and results. The Lagrangian used in this EFT framework is Lorentz invariant, gauge invariant, and CP conserving. Though this Lagrangian has 23 free parameters, it is possible to determine all these parameters simultaneously. The Higgs couplings can be extracted in a highly model-independent way in the sense that all models of new physics are describable either by the addition of local operators to the SMEFT or by the addition 


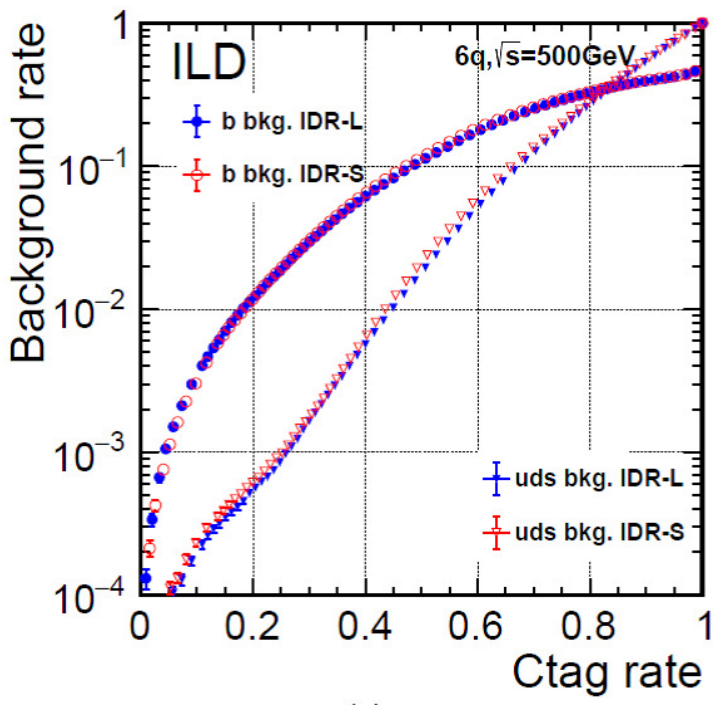

(a)

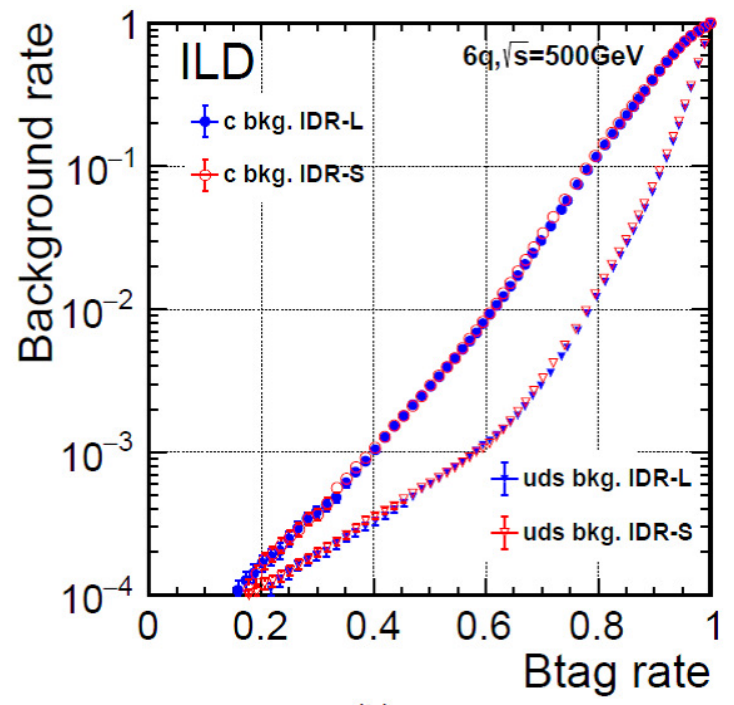

(b)

Figure 1: Flavour tag performance for the large and small ILD detector models. (a) background rate as a function of the $c$-tagging efficiency for $b$-quark and light flavour quark jets. (b) background rate as a function of the $b$-tagging efficiency for $c$-quark and light flavour quark jets. Taken from Ref. [9].

of invisible and exotic Higgs decays. In the global fitting, not only the statistical uncertainties but also systematic uncertainties have been considered [1]. Figure 3 shows the results of global fitting. Already at $\sqrt{s}=250 \mathrm{GeV}$ stage of the ILC, many couplings are reached to $\sim 1 \%$ precision in combination with the HL-LHC results.

In Figure 4, a comparison has been made between polarized and unpolarized beams. When we compare the results of " $2 \mathrm{ab}^{-1} 250 \mathrm{GeV}$ polarized" and of " $5 \mathrm{ab}^{-1} 250 \mathrm{GeV}$ unpolarized", there are no drastic differences. In general, higher statistics always help to improve precision. However, beam polarization allows us to have more independent measurements and have better control of systematics. These facts give us more constraints in the global fitting, which is another factor of the improvement. The beam polarization is a very powerful tool, essentially compensates for a factor of 2.5 in integrated luminosity.

\section{Summary}

In this contribution, we have discussed the Higgs physics potential at the ILC with a few examples. It is possible to determine the Higgs couplings in a highly model-independent way under the EFT framework at the ILC, and most of the couplings can be measured within $\sim 1 \%$ precision even at $\sqrt{s}=250 \mathrm{GeV}$ stage. The beam polarization is a very powerful tool, essentially compensates a factor of $\sim 2.5$ in integrated luminosity. A comparison has been made for the prospects of combination with HL-LHC, illustrating the synergy between projects.

\section{References}

[1] Philip Bambade et al., The International Linear Collider - A Global Project, arXiv:1903.01629 (2019) 

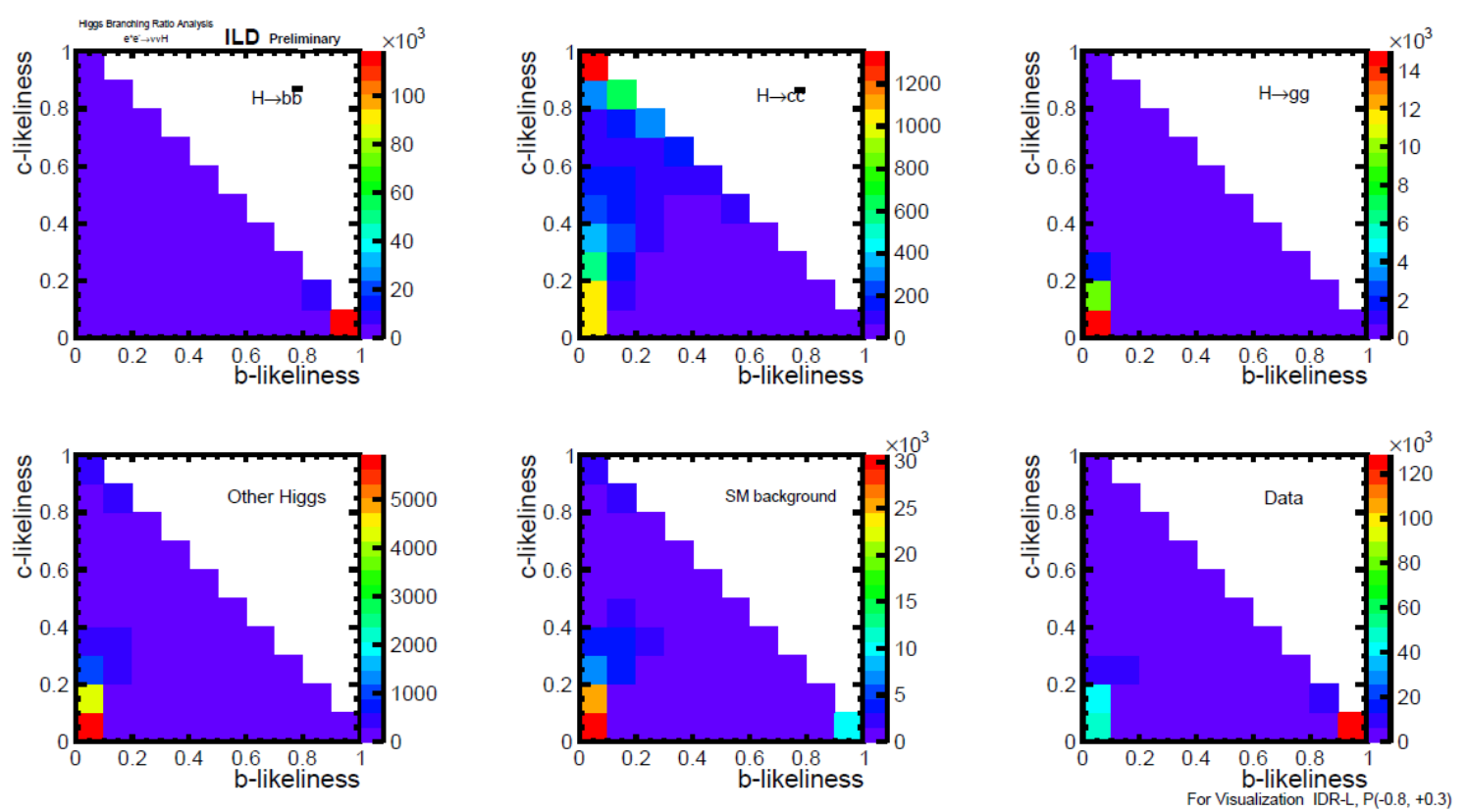

Figure 2: Visualization of the flavor tag performance in $e^{+} e^{-} \rightarrow v \bar{v} h$ process at $\sqrt{s}=500 \mathrm{GeV}$. The panels show the 2D distributions of $c$ - versus $b$-likeliness separately for $h \rightarrow b \bar{b}, h \rightarrow c \bar{c}, h \rightarrow g g, h \rightarrow$ other, the SM background and their mix expected in the template sample. Taken from Ref. [9].

[2] Ties Behnke et al., The International Linear Collider Technical Design Report Volume 4: Detectors, arXiv:1306:6329 (2013)

[3] J. Yan et al., Measurement of the Higgs boson mass and $e^{+} e^{-} \rightarrow Z H$ cross section using $Z \rightarrow \mu^{+} \mu^{-}$and $Z \rightarrow e^{+} e^{-}$at the ILC, Phys. Rev. D 94, 113002 (2016)

[4] M. A. Thomson, Model-independent measurement of the $e^{+} e^{-} \rightarrow H Z$ cross section at a future $e^{+} e^{-}$linear collider using hadronic $Z$ decays, Eur. Phys. J. C (2016) 76:72

[5] LCC Physics Working Group, Physics Case for the International Linear Collider, arXiv:1506.05992 (2015)

[6] ILC Parameters Joint Working Group, ILC Operating Scenario, arXiv:1506.07830 (2015)

[7] LCC Physics Working Group, Physics Case for the $250 \mathrm{GeV}$ Stage of the International Linear Collider, arXiv:1710.07621 (2017)

[8] T. Suehara and T. Tanabe, LCFIPlus: A Framework for Jet Analysis in Linear Collider Studies, Nucl. Instrum. Meth. A808, 109 (2016),

[9] The ILD Concept Group, International Large Detector Interim Design Report, arXiv:2003.01116 (2020)

[10] Tim Barklow et al., Improved formalism for precision Higgs coupling fits, Phys. Rev. D 97, 053003 (2018) 


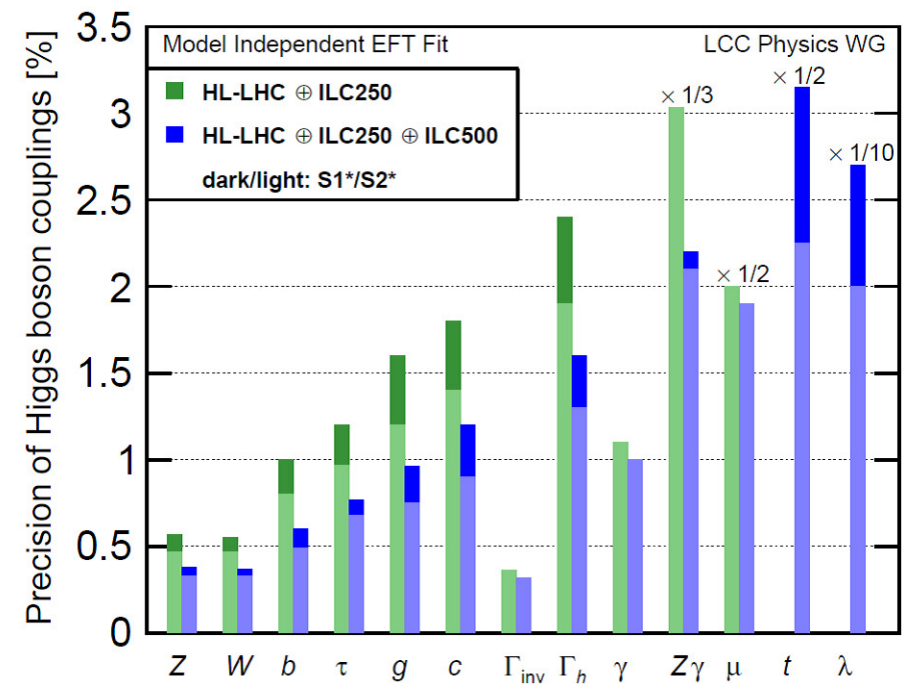

Figure 3: Projected Higgs boson coupling uncertainties for the ILC program at $\sqrt{s}=250 \mathrm{GeV}$ and an energy upgrade to $\sqrt{s}=500 \mathrm{GeV}$, using the SMEFT framework. S1* is the results based on current full simulation, and S2* is the expected results assuming improvements in analysis techniques and tools. Taken from Ref. [1].

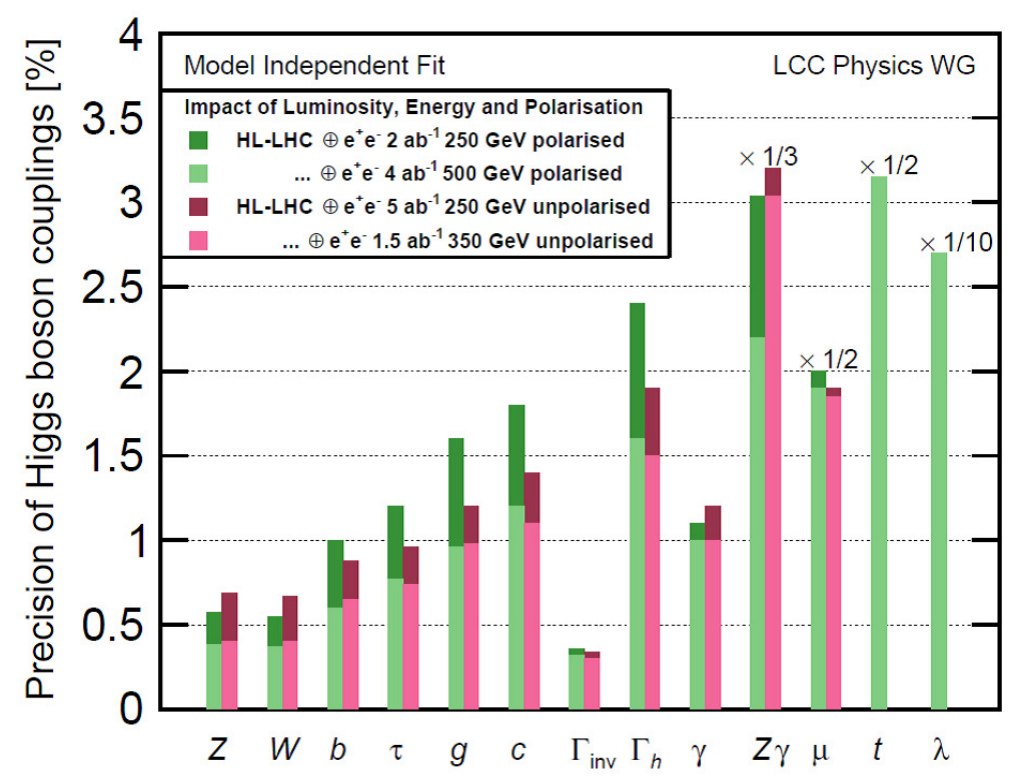

Figure 4: Projected Higgs boson coupling uncertainties obtained under the SMEFT framework for different integrated luminosities, for polarized/unpolarized beams. Taken from Ref. [1].

[11] Tim Barklow et al., Model-independent determination of the triple Higgs coupling at $e^{+} e^{-}$ colliders, Phys. Rev. D 97, 053004 (2018) 